\title{
Visualization of Personal History for Video Navigation
}

\author{
Abir Al-Hajri, Gregor Miller, Matthew Fong, and Sidney Fels \\ Human Communication Technologies Laboratory, University of British Columbia, Vancouver, BC, Canada \\ \{abira, gregor, mfong, ssfels\}@ece.ubc.ca
}
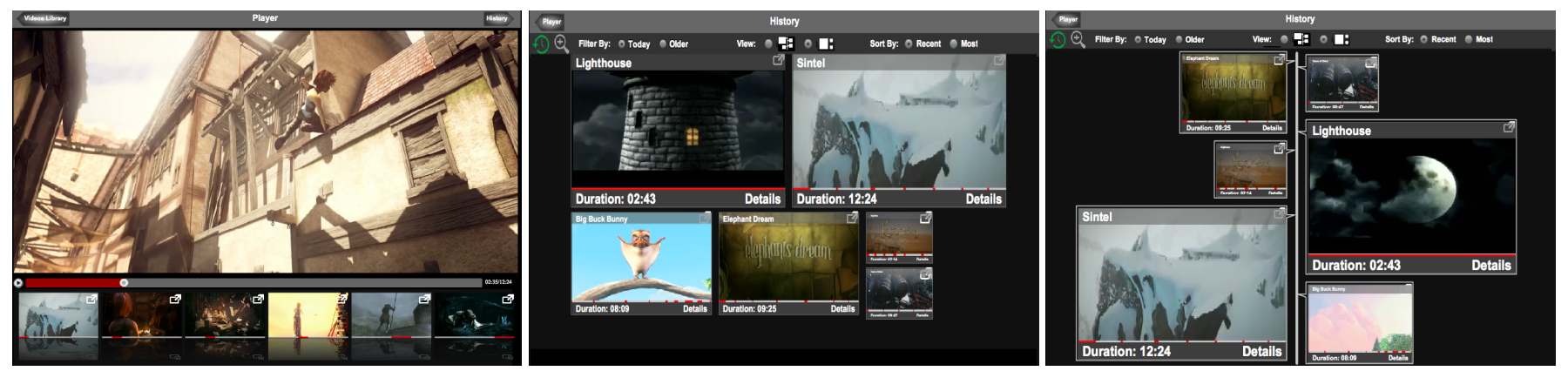

Figure 1. Our prototype history-based interface called the Video History System (VHS) aids navigation through the management of a user's personal viewing history. Playback of video is controlled with familiar tools such as play/pause, seek and filmstrip (left) - the VHS records each part of the video viewed by the user. The history is then visualized in one of two ways: as Video Tiles (centre) or as a Video Timeline (right). ${ }^{1}$

\begin{abstract}
We present an investigation of two different visualizations of video history: Video Timeline and Video Tiles. Video Timeline extends the commonly employed list-based visualization for navigation history by applying size to indicate heuristics and occupying the full screen with a two-sided timeline. Video Tiles visualizes history items in a grid-based layout by following pre-defined templates based on items' heuristics and ordering, utilizing screen space more effectively at the expense of a clearer temporal location. The visualizations are compared against the state-of-the-art method (a filmstrip-based visualization), with ten participants tasked with sharing their previously-seen affective intervals. Our study shows that our visualizations are perceived as intuitive and both outperform and are strongly preferred to the current method. Based on these results, Video Timeline and Video Tiles provide an effective addition to video viewers to help manage the growing quantity of video. They provide users with insight into their navigation patterns, allowing them to quickly find previouslyseen intervals, leading to efficient clip sharing, simpler authoring and video summarization.
\end{abstract}

\section{Author Keywords}

Video; Navigation; History; Visualization

\section{ACM Classification Keywords}

H.5.2. Information Interfaces and Presentation: User Interfaces; H.1.2. Models and Principles: User/Machine Systems

Permission to make digital or hard copies of all or part of this work for personal or classroom use is granted without fee provided that copies are not made or distributed for profit or commercial advantage and that copies bear this notice and the full citation on the first page. Copyrights for components of this work owned by others than the author(s) must be honored. Abstracting with credit is permitted. To copy otherwise, or republish, to post on servers or to redistribute to lists, requires prior specific permission and/or a fee. Request permissions from Permissions@ acm.org.

CHI'14, April 26-May 1, 2014, Toronto, Canada.

Copyright is held by the owner/author(s). Publication rights licensed to ACM.

ACM 978-1-4503-2473-1/14/04\$15.00.

http://dx.doi.org/10.1145/2556288.2557106

\section{INTRODUCTION}

Watching videos is a major online activity, accounting for a large proportion of time spent online: according to comScore $^{2}, 187$ million people in the U.S.A. watched more than 48 billion online videos in July 2013. 100 hours of video are uploaded to YouTube ${ }^{\mathrm{TM}}$ every minute, over 6 billion hours of video are viewed a month and $68 \%$ of people who watch online videos share them with friends ${ }^{3}$. 500 years of YouTube video are watched every day on Facebook, and over 700 YouTube videos are shared on Twitter every minute ${ }^{3}$. Given the volume of available video, new interfaces are required to help manage and manipulate video spaces. Existing interfaces and services have not addressed problems managing previously seen video, and using this data allows for novel and improved applications. We present an investigation into two visualizations of personal video navigation history, to determine how users behave when given a history and if they feel is beneficial to the state-of-the-art methods.

Video navigation histories are a simple archive that a person can use to easily find a previously viewed video interval. They may navigate to the exact location within the original video simply by clicking on the references within their history. This provides the user with a record for historical navigation and removes much of the burden of relying on memory. This history offers users multiple applications such as video chaptering, monitoring their own navigation behaviour, re-watching clips, saving clips that can then be used for sharing, videos summarization, or highlights creation. However, finding previously viewed content in a navigation history is

\footnotetext{
${ }^{1}$ Video and screenshots licenced under Creative Commons Attribution 3.0, (c) 2006 - 2012, Blender Foundation

2 www.comscore.com/Insights/Press_Releases/2013/ 8/comscore_Releases_July_2013_U.S._Online_Video_ Rankings

3 www.youtube.com/t/press_statistics
} 
often a difficult task due to the design, organization, and volume of information to visualize. This issue has received significant attention within the context of web browsing histories, but little attention has been paid to video navigation history. Aside from the commonly employed list-based representation of the visited videos in a reverse chronological order (e.g. YouTube, Netflix), to our knowledge, our previous work [1] was the first that provided a detailed visualization of a single video navigation history. Thus, the goal of this research is to extend the work for multiple-video history by testing different design layouts that support user-centred management of history, and evaluate the benefits this brings.

The benefits of a navigation history come from two user behaviours. First, users do not always watch a video straight through, but may skip parts (they view only intervals within the video) - this is one of the uses of the seek mechanism, for example. (Even if users watch all videos without interruption, our interface effectively presents a recently viewed videos list, much like YouTube currently does.) Second, an emerging behaviour is that users re-watch parts of the video, allowing visualizations to present additional information such as view count or provide estimates of the location of affective intervals within the video. The fact that re-watching behaviour is becoming more common is not so obvious or intuitive; however, it has been shown that users re-watch segments of video as part of the contemporary browsing and navigation experience, for re-experiencing and enjoying favourite parts or to discover subtle details which were previously missed such as with instructional videos [17]. Studies have also shown that videos containing certain emotional triggers, such as comedy or personal attachment, cause repetitive watching [22, 18]. Thus, a personal navigation history may improve the user experience for these use cases.

To illustrate this behaviour and how a visualization for a video history can be utilized, a usage scenario is presented below.

As a university student, Nick watches a lot of course lectures along with other online videos. As he watches a video, he frequently jumps around the video trying to understand the content or to find answers to specific questions. These jumps (seeks) on the video are captured on his history which is available to him at anytime. This allows him to favourite the intervals he thinks are important to note, delete the clips that are not worth keeping, or just browse his history to recap the lectures.

For his university courses, he sometimes goes back to a specific lecture to find answers for his assignments. He could either use his history if he has seen the clip that contains the information, or if he has time he could navigate the video again. If he decides to look at his history trying to find the answer, he could easily look for the lecture title or thumbnail from his history. Accessing the details of that lecture would give him a full history of how he consumed that video and all the portions he watched. The viewing history of the lectures would be full of unnecessary segments if he had not cleaned or managed his history. However, using the provided filters he could find the parts or segments that he has watched multiple times as well as the marked 'favourite' ones. Moreover, if he remembers roughly where the intended information is located within the video timeline, then he can sort the events based on their temporal location within the video. The intended clip can then be easily viewed, saved or shared by clicking on the corresponding thumbnail.

To offer such accessibility described in the above scenario, we propose two visualizations of a detailed multiple-video navigation history: Video Tiles and Video Timeline. These are both part of our Video History System (VHS) framework, and utilize the same underlying representation. A user study which compares the history visualizations to an interface without access to a detailed history is presented; results demonstrate that searching for and sharing personal affective intervals using the proposed visualizations is faster and preferred by participants.

\section{RELATED WORK}

The human visual system can perceive graphical information such as pictures, videos and charts in parallel, however, text can only be perceived sequentially [7], since the human brain processes visual input earlier than textual input [19]. Nadeem et al. showed that the use of visual aids in history mechanisms is more effective than the use of only textual data [16], thus having a visualization of the video navigation history can help extensively when searching for information. Nadeem $e t$ $a l$. also demonstrated that the use of history mechanisms may have a significant effect on user satisfaction and performance when revisiting previously viewed content. These findings reveal that it is important to develop and enhance history visualization mechanisms. However, since there is little work on historical video navigation, we are going to explore this from the perspective of web browsing history.

In most web browsers, history is represented as a list of the visited web pages' titles sorted by date, popularity or aggregated by some time period. The history menu opens in a new window where pages are visualized as titles or with thumbnail images. Researchers have tried various visualizations to simplify searching within the history, which can be divided into three categories: timeline-based, graph-based and 3D.

In the timeline-based visualization, history consists of a linear scrollable list of thumbnails, which appear in reverse chronological order: the most recently visited page is at the top of the list, and clicking on any of these thumbnails (or icons) redirects the user to the corresponding web page. Most web browsers, YouTube, Netflix, Hodgkinson [8] and Girgensohn et al. [6] use this visualization to represent the history of user's visited content as shown in Figure 2(a), where clicking on one of the thumbnails navigates to the corresponding content. Li et al. [11] and Hupp et al. [9] used this approach for their detailed histories with the addition of a list of coloured icons next to each thumbnail describing the user's performed actions. However, this visualization faces problems when multiple tabs or multiple browsers are opened at the same time. Vartiainen et al. [20] developed the Rolling History for mobile devices where they proposed four directions of navigation control: instead of having one reel of thumbnails, they used two - horizontal and vertical, shown in Figure 2(b). All opened browsers are in the vertical reel; 


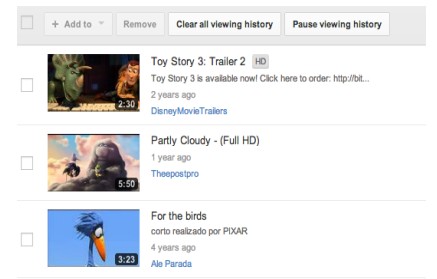

(a) YouTube $\mathrm{TM}$

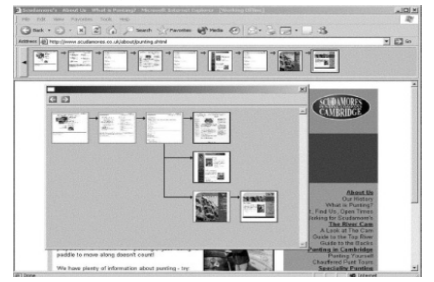

(e) Tree (Directed Graph)

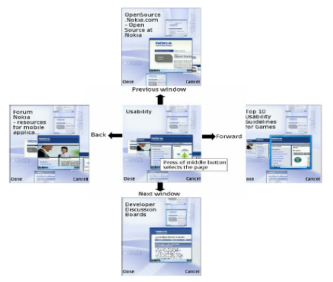

(b) Rolling History

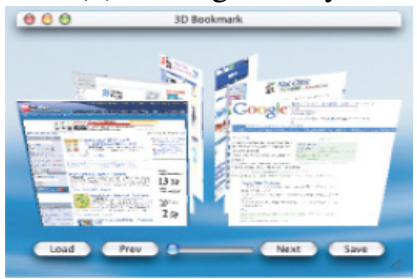

(f)WebBook

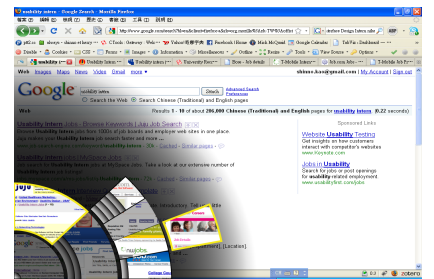

(c) TabViz

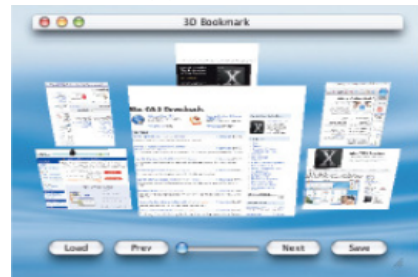

(g) Circle

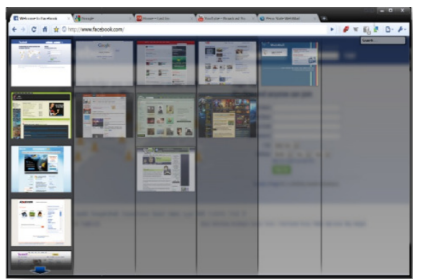

(d) Grid of Thumbnails

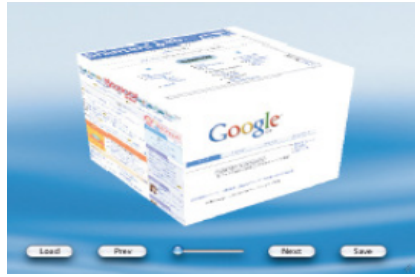

(h) Cube

Figure 2. Previous work on video or web histories can be divided into three categories of visualization: (1) Timeline based: YouTube's history consisted of a list of thumbnails ordered chronologically; Rolling History [20] used four directions of navigation control to cover the history of multiple tabs or browsers opened at the same time; $\mathrm{TabViz}^{4}$ employed a fan-shaped hierarchical visualization to show the history of multiple tabs; Grid of thumbnails [10] displayed the history of each tab in a vertical column of the grid; (2) Graph-based: Tree or directed graph [14] visualized each visited page as a node and links as the edges between nodes; (3) 3D visualization: WebBook [21] represented each web page as a traditional book page; Circle mode [21] placed thumbnails of the visited web pages at the circumference of a circle; $C u b e$ mode [21] placed thumbnails of pages on the faces of a cube.

the currently active browser appears in the middle, and its history is visualized in the horizontal reel. Blankenship et al. proposed $\mathrm{TabViz}^{4}$, which uses a fan-shaped browser tab visualization where concurrent active tabs are represented in a radial hierarchical structure, visualizing the parent of each opened tab (Figure 2(c)). Khaksari proposed a Grid as another solution for multiple tabs opened at the same time [10]. The Grid consists of a number of labeled tabs, where each tab corresponds to the relevant tab in the browser. Each vertical column of thumbnails is mapped to the history of corresponding tabs in the background (shown in Figure 2(d)). According to the article this visualization reduces cognitive workload, increases enjoyability and reduces user frustration.

Milic-Frayling et al. [14] deduced that high effectiveness during search requires users to have a mental map of both the hierarchical structure and the access sequence of web pages. Using the timeline-based visualization, back-tracking or visiting new content from the currently viewed history item would affect the structure of the history, creating confusion and affecting the searching task. This can be solved using a 2D graph-based visualization where the history is presented as a horizontal tree (e.g. $[14,15])$. A new branch is generated from the parent node whenever a user back-traces and visits a new page, as shown in Figure 2(e). Mayer [12] used a directed graph where each visited page is a node and the edge between them is the link. Pages that are visited multiple times are visualized using a single node to avoid repetition. Mayer used the size of the node to represent the time spent on the corresponding web page.

The final category is the use of 3D visualizations for browsing history. Frecon et al. [5] developed WEBPATH, which visualizes the graph of the user's browsing history using a 3D representation. Each visited page is a cube labeled with the

\footnotetext{
${ }^{4}$ http://tabviz.org/
}

page title on top and the surface of the cube shows an extracted image from the HTML description of the page. Users have control over which image can be used to represent the page. This visualization has not been evaluated to check its usability and performance. Card et al. proposed WebBook [2], which used a book metaphor to aggregate web pages in virtual 3D books. A web page is represented as a traditional page in a WebBook as shown in Figure 2(f). However, in terms of search speed, this design might not perform well due to the need for flipping or visiting most of the pages that precede the desired page. Yamaguchi et al. also proposed two other visualizations: a circle mode and a cube mode as shown in Figure $2(\mathrm{~g})$ and $(\mathrm{h})$ respectively. In a circle layout, the thumbnails of web pages are placed around the circumference of a circle while in the cube layout thumbnails are put on the surfaces of cube. However, it is not clear how scalable the cube is, since the surface area is limited.

None of the approaches proposed to date have been evaluated or used to visualize a detailed video navigation history aside from our recent work [1]. Research within video history (e.g. $[6,15]$ and YouTube) used the aforementioned approaches only to visualize the previously watched videos but not how these videos were navigated (i.e. intervals). However, Al-Hajri et al. [1] visualized a single video history as a list of interactive segments, whereas, in this paper we are looking at multiple-videos navigation history. In this research, we suggest and evaluate two other visualizations for a detailed video navigation history and investigate which layout improves search efficiency and user preference.

\section{VIDEO HISTORY SYSTEM (VHS)}

The goal of this work is to provide users with intuitive access and management of their personal video viewing history. This requires an interface that can capture and visualize the detailed navigation history of the videos viewed by a user. To 


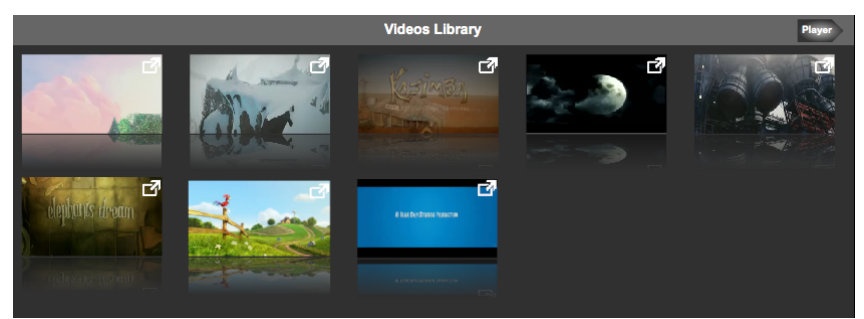

Figure 3. The video library provides a grid of videos from which users may select the one they would like to view. Each video is represented as a thumbnail containing a small video preview; clicking on any video will begin playback in the main player. As part of our interface, we consider this analogous to the results of a search, or as a set of suggested content.

be able to test our proposed visualizations, we need an interface to be quite functional to support realistic video watching experiences comparable to commercial systems so that the visualizations are integrated with the same fidelity. Thus, due to the lack of such interfaces we developed a prototype viewer, we called Video History System (VHS), for evaluating history visualizations. In this section we give a detailed description of this interface, how the history is captured, the different visualizations designed for the history, and the scalability of the visualizations.

\section{History Record Representation Structure}

The principle goal of our interface is to provide efficient access to previously viewed videos, and in particular, the intervals viewed within those videos. We accomplish this by applying our approach, described in [1], where a continuous video history is recorded for the user as they consume video. Using the video navigation interface described below, navigation-level events are captured by recording high-level user actions such as seek, play, pause, and changing video. Each time a user watches a video segment, a new entry is added to the history record. It records the video segment using a unique ID for each video along with the segment start and end time. An accumulated view count for every instant of time in all viewed videos is calculated from the intersection of all viewed intervals for each video. This provides the user with a better understanding of the importance of each interval based on their viewing frequencies. This can be used later to facilitate fast navigation and search.

\section{Video Navigation Interface}

The interface is based on three modes: the video library, the viewer and the history. Users can easily switch between modes using the navigation controller. To choose a video, users can either use the video library shown in Figure 3 or they can load a video from disk. Once users select a video to view, the interface transitions to the viewer.

The viewer represents the core component of the interface, allowing users to watch video while their history is captured. The viewer's video player (similar to YouTube, QuickTime, etc.), shown in Figure 4, contains: play/pause via a button (bottom left) or by clicking directly on the video; seek via playhead (white circle) or mouse click on the timeline (red/grey bar); a current playhead time label (white text, bottom right). The viewer also contains a filmstrip, shown under

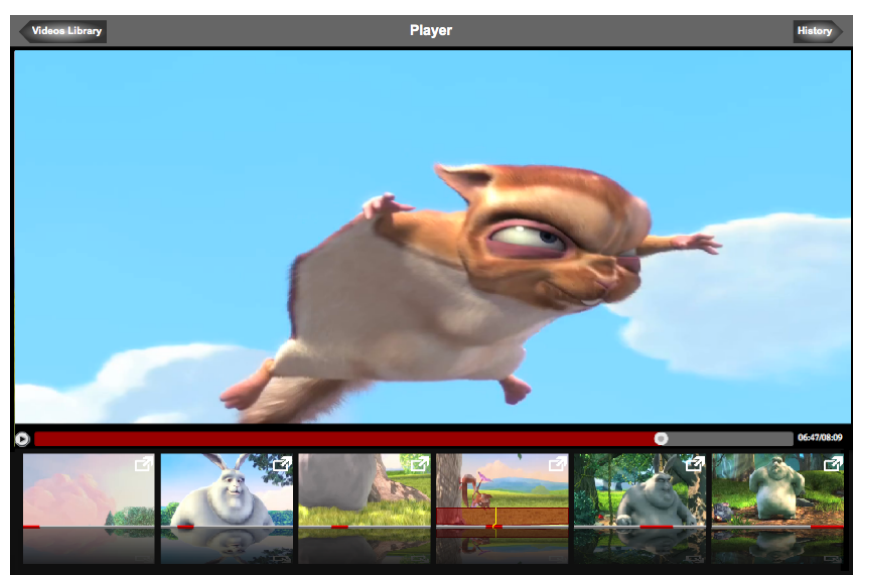

Figure 4. Video playback is performed and controlled within the viewer. The video player occupies the majority of the space; the video can be played/paused using the dedicated button below the player (on the left) or by clicking on the video itself; seeking is controlled via the white circle playhead or simply by clicking/dragging on the red/gray video timeline. The filmstrip below the player provides real-time previews based on the cursor position, allowing faster navigation of the current video.

the player, which consists of a fixed number of thumbnails sampled from the video. The entire video is divided into $n$ equal length intervals ( $n=6$ for our interface), where each interval is represented by a single video segment visualized by the starting frame of that interval. We applied this design since thumbnails are an accepted form of preview in nearly all digital video retrieval interfaces. Moreover, the filmstrip metaphor is commonly used to present content of video as a navigation device, and is considered effective on desktop systems [3], while also providing a quick summary. As such, we chose to employ the filmstrip metaphor to aid video navigation within the viewer. The filmstrip supports level-of-detail manipulation via zooming using mouse wheel gestures with the cursor over the target segment.

Each video segment, shown in Figure 5 (right), can be individually searched (via seek) and played to minimize the time needed to search for a specific event. Moving the cursor over the lower portion of the segment pops up a high-resolution visualization of the interval's timeline which can be used to seek within the video interval using mouse motion as a cue. The seek point is communicated by a yellow line and the thumbnail image updates to reflect the current seek position. To play the interval in the main player, users can drag the seek location in the segment to the white square (with an arrow) at the top right corner.

Every time a user seeks or plays a different video/segment within the main player, a new record is added to their history. The user can switch to the history shown in Figure 6 by clicking on the 'History' button: each record is visualized as a video segment with a size indicating how often that interval was viewed. Previous work applied varying colour intensities to indicate the importance of each segment [13]. We used size since we are representing intervals using thumbnails where colour discrimination would be confused with the thumbnail content and would be difficult to differentiate for some videos. 


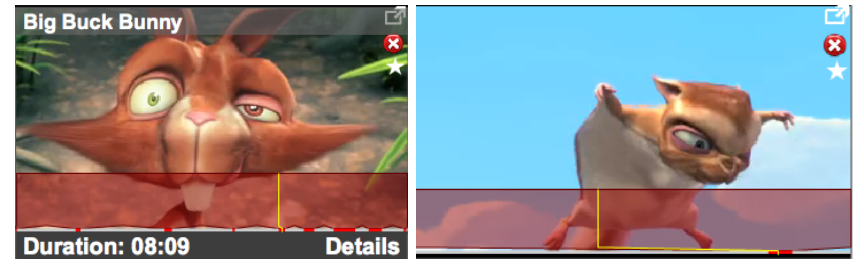

Figure 5. The user's history is visualized as a set of small video segments. An intra-video segment (left) is used to visualize an aggregated history of a single video as one thumbnail, where the union of its segments are visualized in the thumbnail timeline (red/gray bar) and the combined segment has a single seek bar. The single video segment (right) represents a single interval from the history, and is the furthest possible zoom level.

Segment size is determined based on a weight factor which is derived from how often the segment has been viewed as well as its duration. For our study we used a weight factor of $75 \%$ of the peak view count plus $25 \%$ of the duration in minutes (normalized duration is not used to ensure short segments are not biased to a larger size). We define three different sizes for segments within our interface based on this weight factor: small, medium and large. Thumbnail sizes are proportional to each other such that they fit the tile template patterns; for our interface, we use $320 \times 180$ (large), $210 \times 118$ (medium) and $100 \times 56$ (small), measured in pixels on a one-to-one PPI display.

The history visualizations contain an inter-video history, which shows the different videos the user viewed, with all intervals watched within a single video aggregated into an intra-video segment (Figure 5, left). The union of the viewed intervals of a single video is easily visualized in the timeline of the video segment representing the accessed video in the history. To access the detailed history of a specific video, the user can click on the 'Details' link, which opens a detailed history of the selected video only. The detailed history has a similar structure to the history mode with the exception of the removal of the Details info for the detailed history video segment as shown in Figure 5 (right).

\section{History Visualization Designs}

The main goal of this paper is to provide users with a history management tool via an effective visualization. Based on a review of the literature, existing interfaces and services, we made various design decisions to create a visualization of the complete history of a user's video navigation. Since the list (Figure 2(a)) and grid (Figure 2(d)) layouts are the most commonly used visualization for browsing history, we decided to apply these layouts within our interface due to their familiarity with users and their applicability to video visualizations. We intend to investigate how users would employ these layouts for video history and how they could accomplish their tasks compared to the previous design.

From our pilot study (described in the evaluation section) investigating the time taken to search for events, users with a history and our history visualizations performed faster than when compared to without a history, but these results were not significant. However, users' comments indicated a strong liking (6.3 on a 7-point Likert scale) for the interface and commented that they enjoyed it. User feedback inspired us

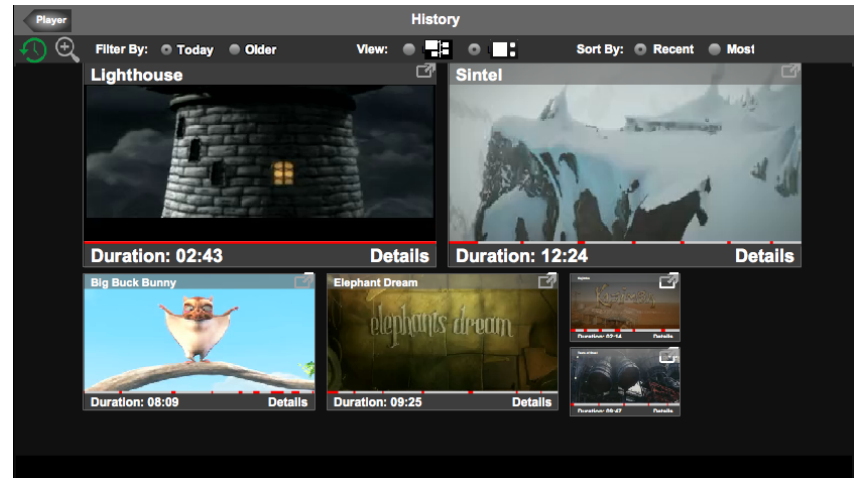

Figure 6. Each history visualization (presented in the History mode) displays the user's navigation history, and provides top-level access to all previously viewed videos from which the user may zoom into any history entry for more detail. The history can be filtered by date, sorted by time or popularity, and the type of visualization used can be chosen. The thumbnails' size is based on the view count within each video.

to extend the list and grid interfaces by utilizing the size of thumbnails for heuristics (in both) and expanding the list to occupy a full screen (by using two lists). Our refinement ended with two novel visualizations: Video Timeline and Video Tiles, as described below. These visualizations were tested to evaluate their performance when performing a search task, while also measuring user satisfaction.

\section{Video Timeline}

This visualization of video history was an extension to the familiar list visualization, with the exception of having two columns of variable-sized thumbnails instead of just one. It is designed to display a significant number of thumbnails within a small area, while also maintaining an explicit order, by dividing them into two columns along the user's vertical timeline.

For users to relate these thumbnails to their occurrence in time, they are attached to the user's vertical timeline, where the attachment location indicates their order with respect to the other thumbnails in the history, as illustrated in Figure 7 (left). By default the thumbnails are in reverse chronological order (based on user time), however this order can be changed to interval start time (Time, only available in detailed history), recently viewed (Recent), and viewing weight (Most) by using the corresponding radio buttons shown at the top right of the Figure. The interface also supports filtering the user's history based on favourite segments and/or intervals that have been watched twice or more (these features are available in both visualizations).

\section{Video Tiles}

This history visualization was designed to take advantage of the entire screen space, using an implicit ordering. This design is essentially a grid layout of thumbnails, which is commonly used for web browsing history with the exception of having varied sizes for the thumbnails in our design. Moreover, in our design the grid represents a detailed video history where each grid cell corresponds to an interval within a video. Using this design allows us to display more thumb- 

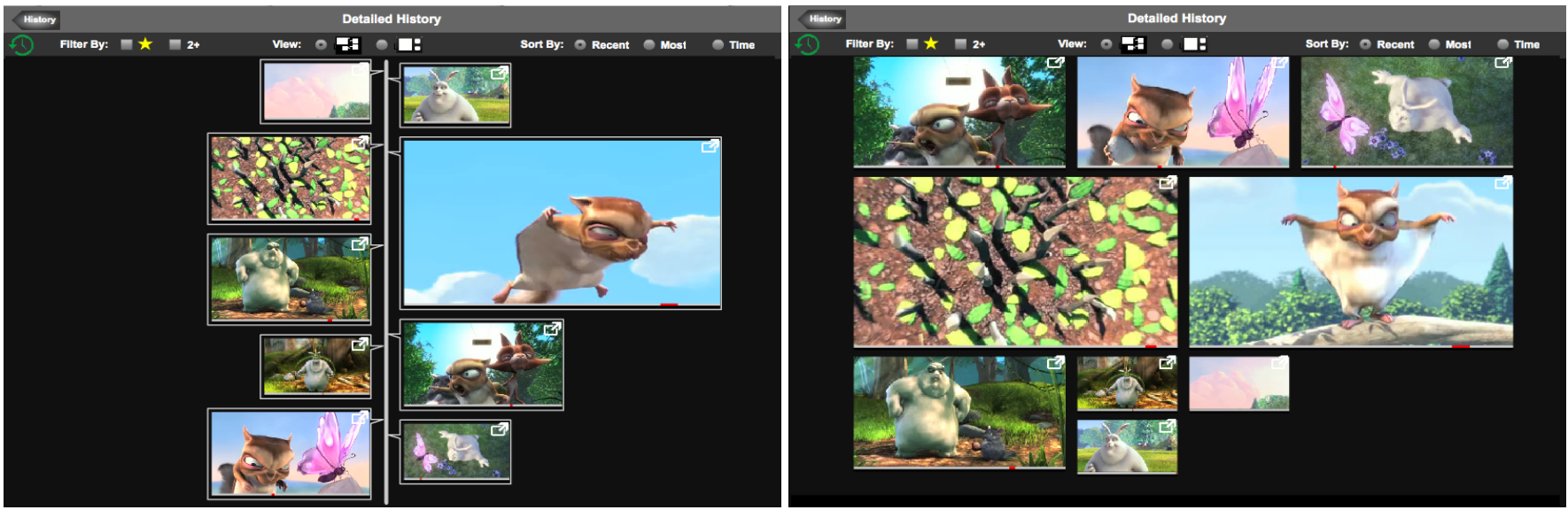

Figure 7. We propose and investigate two history visualization designs: Timeline (left) attaches history segments to a user's vertical navigation timeline using two columns; Tiles (right) displays history segments based on template patterns following Algorithm 1, based on their view count and chosen order. Both use VHS-based heuristics to choose the size of thumbnails and offer level-of-detail control via "zooming" into individual segments.

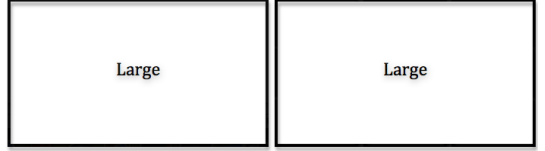

Pattern 0

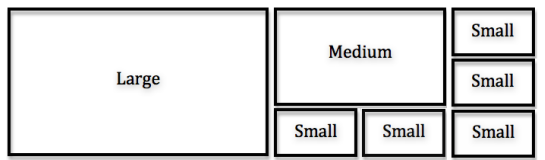

Pattern 1

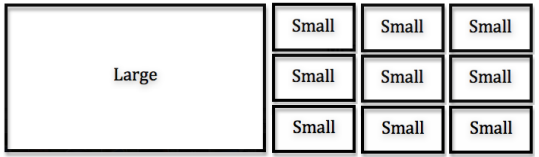

Pattern 2

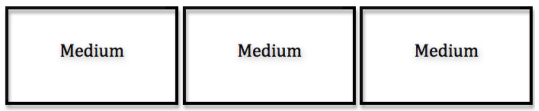

Pattern 3

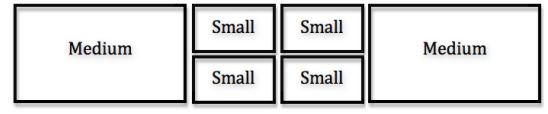

Pattern 4

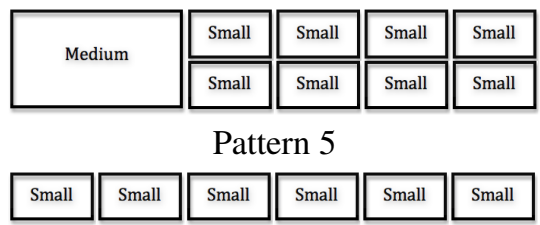

Pattern 6

Figure 8. Template patterns are used for the Video Tiles visualization, to provide a clean set of thumbnails with an implicit order (top-to-bottom, left-to-right, based on a single pattern). Patterns 1, 2, 4, and 5 have alternatives where either the entire pattern is reflected or just the portion containing medium and small tiles: Pattern 1 has 8; Pattern 2 has 4; Pattern 4 has 6; Pattern 5 has 5 . These are applied using Algorithm 1.

nails at once with less scrolling needed when searching for video segments.

In our design shown in Figure 7 (right) we used seven different templates (Figure 8), where the order of the segments and their sizes determines the template to be used and the location of segments within these templates. The template is selected based on the conditions explained in Algorithm 1. Within each template, thumbnails are displayed top-to-bottom and then left-to-right based on their order within history.

\section{Visualization Scalability}

As users view hundreds of videos and interact with them, their history will continue to grow. Clearly our visualizations must scale with the data being recorded. In our visualization, the length of a video does not have an effect on the visualization when it is viewed passively (i.e. no user interaction) where it is represented by one video segment. However, the number of interactions or seek actions a user performs with any length of a video and the number of videos viewed determines the size of the history. One way to address scalability issues is to keep the size of the visualized history down, by choosing some policy to limit the number of segments visualized on screen at any one time. This leads to the need for features and interaction techniques to be able to bring the other history segments into view.
Web browsers visualize a user's browsing history by date. For instance, Google Chrome uses a fixed number of data entries to be visualized per page starting from the last visited URL arranged by date with the option of viewing previous history using the 'Older' link. When 'Older' is activated, three other options are provided: 'Newest', 'Newer', and 'Older'. Some of these web browsers provide users with filters that can be applied when viewing their browsing history, for example, by date, name, most frequently visited, and most recently visited. In our visualization for the inter-video history, we follow this approach by limiting the number of visualized items at any time and provide access to the older history items, as shown in Figure 6. For the detailed history visualization, we group close segments in history together as a stack of thumbnails when the number of elements exceeds the limit, as shown in Figure 9. Using the mouse wheel on these stacks, we can zoom in and out, which reveals a new visualization of only that zoomed stack. The front thumbnail of the stack is represented by a seekable and playable video segment while the other thumbnails in the stack are representative images. Our visualization also supports filtering by most frequently viewed, most recently viewed, starting timestamp, favourite intervals, and intervals viewed more than once. 


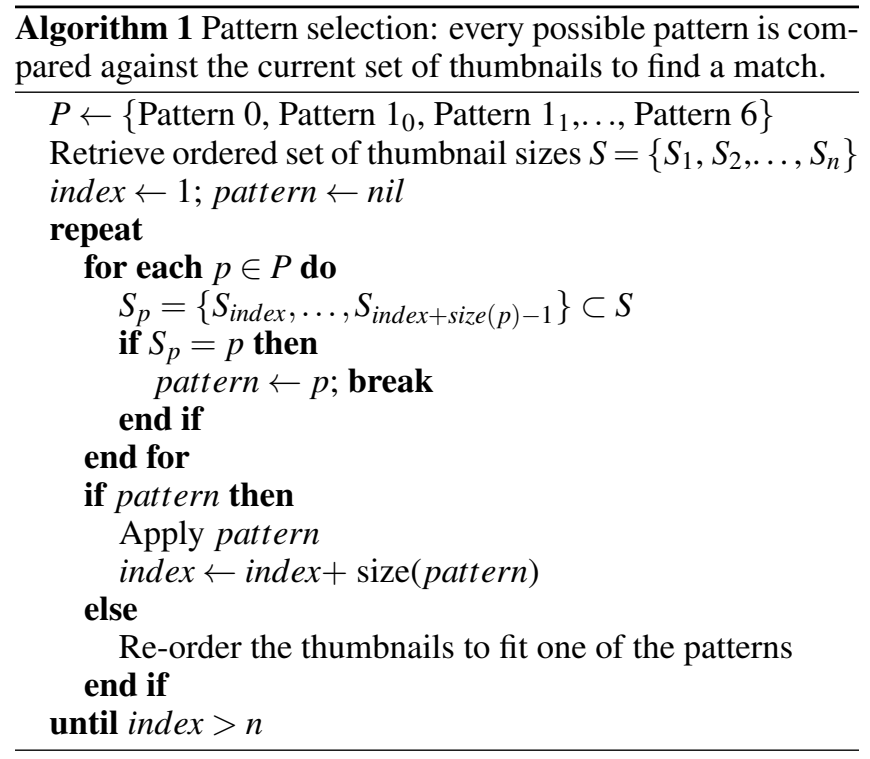

\section{EVALUATION}

A user study was performed to evaluate the different visualization layouts for navigation of video history. Our aim was to investigate which visualization layout would be most efficient when finding previously watched segments within videos, which one would make history navigation easier, and which layout users prefer. Thus, we designed comparative studies where the participants were asked to freely watch a set of videos and then find and share their liked segments from their personal history with two different visualization layouts. We also tested whether these history visualizations performed better than without detailed history where users simply find a video from a grid of previously seen videos (Figure 3) and then search for intervals using the video's filmstrip (similar to what they would have in an online video website such as YouTube).

\section{Design}

Three different visualization layouts were tested. In the pilot test: List, Grid and Filmstrip were investigated, while in the actual study: Video Tiles, Video Timeline, and Filmstrip were experimented. The layout order was alternated between subjects to eliminate the mode order effect. Each participant used the three visualizations to find 7 different clips using each layout. For each clip, participants had to find the corresponding video first using either inter-video history (Figure 6) when one of the proposed visualizations was experimented, or the videos library (Figure 3) when Filmstrip was tested. After finding the video, participants searched for the questioned clip using the provided layout.

Each participant freely watched a set of 5 different short videos of length between 3 and 5 minutes (sourced from YouTube, chosen based on total number of views). They had the option to edit their navigation history by hiding the intervals they did not want to keep using $\boldsymbol{\otimes}$ button in the corresponding video widget or favouriting the intervals they liked using button. They were instructed to keep at least

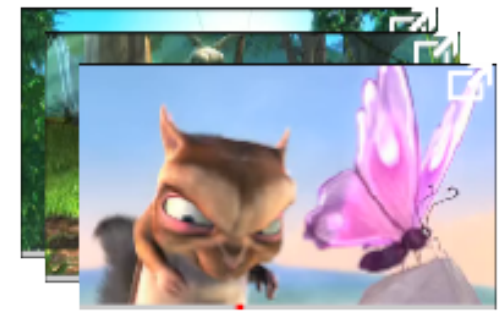

Figure 9. A thumbnail stack is used when the number of segments to be visualized exceeds the limit - this addresses scalability of the history visualization. The user may zoom into or out of the stack via a mouse wheel gesture to obtain control over the presented level-of-detail.

7 segments in the history of each video to be able to start the tasks. Each participant performed 21 search tasks (3 layouts $\times 7$ segments per mode). The participants were asked to find the segments as quickly as possible. For each task, the completion time, the number of previews, and the number of scrolling events were recorded. The completion time was measured once the participant clicked on the Find button until the moment the researcher advanced the task for the participants based on the submitted segments. The navigation heuristics were also recorded during the viewing phase. The participants were asked to rank the different visualization modes based on their speed, easiness and preference, $(1=$ best, $3=$ worst). The experiment lasted approximately one hour per participant.

\section{Pilot Test}

The pilot study was conducted with 5 participants where each subject tried 3 modes to find their previously-seen affective segments. It was carried out to determine user feedback and also, to test the history visualization and the experimental design. In this pilot, two history visualizations, which were chosen due to their familiarity by users viewing videos online and their applicability to what we are trying to visualize, were tested against the Filmstrip. These visualizations are as follows.

\section{List}

The List visualization simply displays the history segments on one vertical strip as shown on the left of Figure 10, where these segments are organized on a reversed chronological order having the last viewed segment at the top of the list. This layout is our previous visualization [1] with the exception of having varied sizes for the thumbnails.

\section{Grid}

History segments in this visualization are organized into $n \times m$ grid of thumbnails as demonstrated on the right of Figure 10, where they are ordered left to right and then top to bottom. The recently viewed segment appears at the most top left corner while the first viewed segment appears at the most bottom right thumbnail. The order of the thumbnails is changeable based on the user preference using the sort options. In this visualization all thumbnails are displayed using a fixed size (medium size), which does not take the viewing weight into account. The fixed size of the thumbnails allowed this visualization to display more thumbnails at a time 

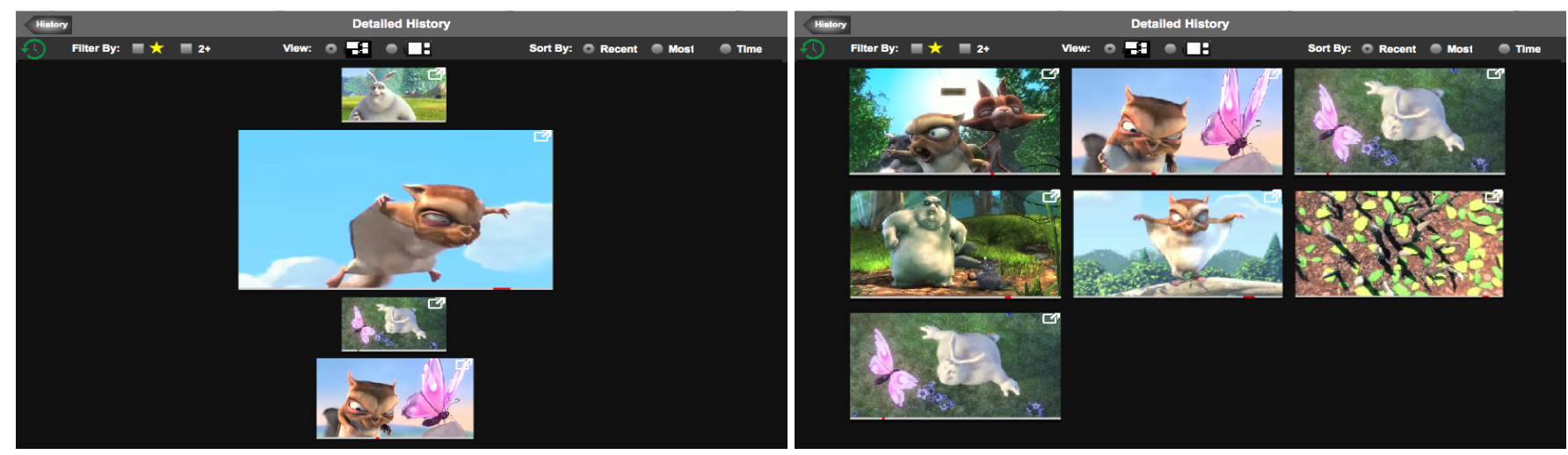

Figure 10. The piloted video history visualization designs: List (on the left ) where history segments are displayed on one vertical scroll-able column, Grid (on the right) where history segments are visualized on an $n \times m$ matrix going from left to right top to bottom.

compared to the other visualization. Thus, less scrolling and searching time are needed for a certain segment.

\section{Results and Lessons Learned from Pilot Test}

The pilot study showed positive results on the features of the interface which encouraged us to continue running the experiment. Most participants commented that they enjoyed their time using the interface and they can imagine seeing its features applied, especially in social networking websites. For the finding task, each participant was able to complete 9 search tasks in less than 40 seconds per segment. Search using the video Filmstrip (no detailed history available) took more time than when having history using the different visualizations. This indicates that having access to the user's personal navigation history leverage finding previously seen interested parts within videos.

Participants ranked Grid as the most liked visualization, then List, and Filmstrip ranked as the least liked mode. However, for the fastest mode they ranked Grid as the fastest, then Filmstrip, while List came last. Some participants mentioned that the List layout needed more scrolling which made them think they were slower than the Filmstrip. However, they liked having different sizes for the thumbnails to indicate how an interval was consumed. This made some participants favour the List visualization over the Grid which is worth investigation.

Based on these pilot results and users feedback, we extended the two visualization layouts as explained in the History Visualization Designs section to utilize different sizes for the Grid layout and including more thumbnails into the List design. To evaluate the new proposed visualization, we run the same experiment but with the new visualizations: Video Timeline and Video Tiles. Moreover, to illustrate the differences between methods, more subjects were recruited for the experiment and more tasks were performed per method. In this experiment, we hypothesized that users would perform better when history is available in terms of time needed to find their previously-seen favourite segments, and that less navigation would be required. In terms of the visualization layouts, we anticipated that the Video Tiles layout would outperform Video Timeline layout for time and scrolling needed since more thumbnails can be viewed at once. However, in terms of seeking or navigation events used, we expected no difference since they both have the same thumbnail representations. For modes preference, we predicted that it would highly depend on participants familiarity with List and Grid layouts.

\section{Participants}

Ten paid volunteers, 6 female and 4 male, participated in the experiment (different from those in the pilot). Participants ranged in ages from 19 to 35 . Each participant worked on the task individually. All participants were experienced computer users and have normal or corrected to normal vision. Seven participants watch online videos on a daily basis and the other three watch videos 3-5 times a week. Five of the participants watch 1-3 videos on average per day, while three watch 3-5 videos per day and two watch more than 10 videos per day.

\section{Results and Discussions}

The study showed strongly positive results for the interface. Each participant was able to complete all 21 search tasks in less than one minute per segment. A one-way, within-subjects ANOVA test analysis was carried out to examine the significance of the results in terms of the average completion time per segment, average number of previews and average number of scrolling events. The results are shown in Table 1. A post-hoc analysis showed that the filmstrip had significantly more previews and took more time than the other two modes, as we hypothesized. This can be explained by the fact that when searching using personal history, participants had a defined context and mental map of their segments [14]. Moreover, when analyzing the participants' navigation history, we found that participants created 11 history segments on average per video. This means that when using history, they had to search for their segment among 11 different thumbnails, whereas when using the video's filmstrip they had only 6 different segments. Despite this, they performed even better when using history as illustrated by the results confirming that detailed history helped them completing the task.

For the history visualization layouts, Video Timeline method was not significantly different from Video Tiles method in both the average number of previews and the completion time. The non-significant results of the completion time might be due to the confusion caused by the flow of the Video 


\begin{tabular}{lccccccccc}
\hline & \multicolumn{3}{c}{ Video Timeline } & & \multicolumn{3}{c}{ Video Tiles } & & \multicolumn{2}{c}{ Filmstrip } & \\
\cline { 2 - 3 } & Mean & SD & & Mean & SD & & Mean & SD & F-test \\
\hline Completion Time & 17.76 & 5.28 & & 17.88 & 4.91 & & 24.31 & 10.42 & $23.01^{*}$ \\
No. of Previews & 25.11 & 28.24 & & 23.41 & 21.03 & & 35.53 & 27.56 & $5.03^{*}$ \\
No. of Scrolls & 6.64 & 7.74 & & 1.54 & 3.96 & & - & - & $28.72^{*}$ \\
\hline
\end{tabular}

Table 1. Usage comparisons for the three methods, using the F-test for equality of means. The results demonstrate that history-based search for personal affective intervals is more efficient than search using Filmstrip and the video library. Notes: SD is Standard Deviation; Completion time is measured in seconds; $* p<0.01$.

Tiles mode as discussed below. However, a significant effect was observed on the number of scrolling events, where Video Tiles had significantly less scrolling events than the Video Timeline. This proves the second part of our hypothesis and the third hypothesis. The more history segments (thumbnails) displayed by the visualization at one view (i.e. less interaction to view more thumbnails), the less scroll events occurred and less time was needed for searching. This was anticipated since all the sizes makes the thumbnails content recognizable and easily identified among other thumbnails. The results for the Video Tiles visualization support this conclusion where less scrolling events occurred in comparison to the Video Timeline visualization. Nevertheless, as the history grows, it becomes almost impossible to view all the segments at once without the need for scrolling when different thumbnail sizes are used. If the scrolling event is disabled following [4], then thumbnails size will shrink as the history builds up making the thumbnails difficult to identify. As is known with visualization scalability of any system there is a trade off between size and scrolling or zooming needs.

When participants were asked to rank the different modes for preference, ease and speed, they ranked Video Timeline as the most liked, easiest and fastest visualization, then Filmstrip, and Video Tiles ranked as the least liked mode. However, the quantitative results for the Filmstrip and Video Tiles modes contradict with the participants ranking for speed, where Filmstrip became last quantitatively and second qualitatively. Some participants mentioned that the flow of the thumbnails in Video Tiles mode created some confusion which they thought it made their performance worse which as we can see is not true. Participants pointed out that the vertical timeline indication used in the Video Timeline mode helped them easily visualize the relationship between the thumbnails. Moreover, some participants indicated that since they are familiar with this layout, it was easier for them to recognize the sequence of the thumbnails and understand the flow. This aligned with what we predicted in our last hypothesis. Thus, more exposure time to the Video Tiles visualization might also help in understanding its representation and flow.

In terms of ease and usefulness of the interface components and features, the average ranking across all components and features was 5.82 out of 7 . All features were ranked above 5 except for two items: the easiness of finding previously seen segments using the Video Tiles visualization $(M=4.7)$, and using the zoom in/out functionality $(M=4.3)$. The low ranking for the ease of finding previously seen segments us- ing the Video Tiles visualization was due to the confusion of understanding the layout and the thumbnails sequence as participants commented. For the zoom in/out functionality, some participants pointed out that it was a bit confusing where a small mouse-wheel gesture triggered multiple zoomin events, causing some frustration. This could also explain the low usage of this feature while performing the tasks where only 2 participants used it when searching for events. The problem with this feature can be resolved by reducing the sensitivity of the mouse wheel. Participants appreciated having this functionality which helped them to get a more detailed view of the Filmstrip segments.

In this study we went with a personalized history approach to get better insight on how this would work in direct real world scenarios. The results and the participants' valuable comments motivate us to present our interface to a larger community. Thus, we plan to design a field study where these visualizations can be investigated on a large scale. By deploying the interface on the internet we can gather more data that would help recommend new features for a video navigation interface.

\section{CONCLUSIONS AND FUTURE WORK}

We have presented two new methods, Video Timeline and Video Tiles, to visualize and navigate a video space using a personal video history. These methods could be integrated easily in a video viewer together or individually as demonstrated in our Video History System (VHS). These visualizations are based on observations of web browsing visualizations and the increasingly temporal nature of video navigation. We performed a comparative study based on a use case of fast searching and sharing, and found significant results in favour of the Video Timeline method. The visualizations were positively perceived and showed significantly faster times for finding previously seen parts when history is used. We conclude that visualizing history is a valuable addition to any video navigation interface and the visualizations we have designed are effective and useful.

We intend to investigate different tasks and procedures that can be used to evaluate the video navigation history within a field study. Through the field study, we will further explore the different visualization approaches (e.g. 3D visualizations) and interactions required when using multiple-video navigation history in a less controlled setting to further refine the utility of our approach. Based on our observations from the study and the results in this work, we are encouraged to perform future empirical studies within social website and edu- 
cational contexts to investigate how this could change the way users consume and navigate video.

\section{REFERENCES}

1. Al-Hajri, A., Miller, G., Fels, S., and Fong, M. Video navigation with a personal viewing history. In Human-Computer Interaction - INTERACT 2013, vol. 8119 of Lecture Notes in Computer Science. Springer Berlin Heidelberg, 2013, 352-369.

2. Card, S. K., Robertson, G. G., and York, W. The webbook and the web forager: an information workspace for the world-wide web. In Proceedings of the SIGCHI Conference on Human Factors in Computing Systems, CHI '96, ACM (New York, NY, USA, 1996), 111-ff.

3. Christel, M., and Moraveji, N. Finding the right shots: assessing usability and performance of a digital video library interface. In Proceedings of the 12th annual ACM international conference on Multimedia, MULTIMEDIA '04, ACM (New York, NY, USA, 2004), $732-739$.

4. Cockburn, A., Gutwin, C., and Alexander, J. Faster document navigation with space-filling thumbnails. In Proceedings of the SIGCHI Conference on Human Factors in Computing Systems, CHI '06, ACM (New York, NY, USA, 2006), 1-10.

5. Frecon, E., and Smith, G. Webpath-a three dimensional web history. In Information Visualization, 1998. Proceedings. IEEE Symposium on (oct 1998), 3 -10, 148.

6. Girgensohn, A., Wilcox, L., Shipman, F., and Bly, S. Designing affordances for the navigation of detail-on-demand hypervideo. In Proceedings of the working conference on Advanced visual interfaces, AVI '04, ACM (New York, NY, USA, 2004), 290-297.

7. Hendee, W. R., and Wells, P. N. T., Eds. The Perception of Visual Information. Springer New York, 1997.

8. Hodgkinson, G. Visually Representative Web History Browser. PhD thesis, Massey University, Wellington, may 2007.

9. Hupp, D., and Miller, R. C. Smart bookmarks: automatic retroactive macro recording on the web. In Proceedings of the 20th annual ACM symposium on User interface software and technology, UIST '07, ACM (New York, NY, USA, 2007), 81-90.

10. Khaksari, G. H. Direct manipulation of web browsing history. International Journal of Computer Science and Information Technology 3, 5 (October 2011), 185-192.

11. Li, I., Nichols, J., Lau, T., Drews, C., and Cypher, A. Here's what i did: sharing and reusing web activity with actionshot. In Proceedings of the 28th international conference on Human factors in computing systems, CHI '10, ACM (New York, NY, USA, 2010), 723-732.
12. Mayer, M. Visualizing Web Sessions: Improving Web Browser History by a Better Understanding of Web Page Revisitation and a New Session- and Task-Based, Visual Web History Approach. PhD thesis, Universitt Hamburg, 2007.

13. Mertens, R., Farzan, R., and Brusilovsky, P. Social navigation in web lectures. In Proceedings of the seventeenth conference on Hypertext and hypermedia, HYPERTEXT '06, ACM (New York, NY, USA, 2006), 41-44.

14. Milic-Frayling, N., Sommerer, R., and Rodden, K. Webscout: support for revisitation of web pages within a navigation session. In Web Intelligence, 2003. WI 2003. Proceedings. IEEE/WIC International Conference on (oct. 2003), $689-693$.

15. Miller, G., Fels, S., Finke, M., Motz, W., Eagleston, W., and Eagleston, C. Minidiver: A novel mobile media playback interface for rich video content on an iphonetm. In Proceedings of the 8th International Conference on Entertainment Computing, ICEC '09, Springer-Verlag (Berlin, Heidelberg, 2009), 98-109.

16. Nadeem, T., and Killam, B. A study of three browser history mechanisms for web navigation. In Information Visualisation, 2001. Proceedings. Fifth International Conference on (2001), 13-21.

17. Russell, C. A., and Levy, S. J. The temporal and focal dynamics of volitional reconsumption: A phenomenological investigation of repeated hedonic experiences. Journal of Consumer Research 39, 2 (August 2012), 341-359.

18. Syeda-Mahmood, T., and Ponceleon, D. Learning video browsing behavior and its application in the generation of video previews. In Proceedings of the ninth ACM international conference on Multimedia, MULTIMEDIA '01, ACM (New York, NY, USA, 2001), 119-128.

19. Teelink, S., and Erbacher, R. F. Improving the computer forensic analysis process through visualization. Commun. ACM 49, 2 (Feb. 2006), 71-75.

20. Vartiainen, E., Roto, V., and Kaasalainen, J. Graphical history list with multi-window support on a mobile web browser. In Internet and Web Applications and Services, 2008. ICIW '08. Third International Conference on (june 2008), $121-129$.

21. Yamaguchi, T., Hattori, H., Ito, T., and Shintani, T. On a web browsing support system with $3 \mathrm{~d}$ visualization. In Proceedings of the 13th international World Wide Web conference on Alternate track papers \& posters, WWW Alt. '04, ACM (New York, NY, USA, 2004), 316-317.

22. Yu, B., Ma, W.-Y., Nahrstedt, K., and Zhang, H.-J. Video summarization based on user log enhanced link analysis. In Proceedings of the eleventh ACM international conference on Multimedia, MULTIMEDIA '03, ACM (New York, NY, USA, 2003), 382-391. 